\title{
A new bronchoscopic method to measure airway size
}

\author{
W.V. Dörffel ${ }^{\#}$, I. Fietze ${ }^{\#}$, D. Hentschel ${ }^{\#}$, J. Liebetruth*, Y. Rückert**, P. Rogalla ${ }^{+}$, \\ K-D. Wernecke , G. Baumann ${ }^{\#}$, C. Witt
}

A new bronchoscopic method to measure airway size. W.V. Dörffel, I. Fietze, D. Hentschel, J. Liebetruth, Y. Rückert, P. Rogalla, K-D. Wernecke, G. Baumann, C. Witt. C) ERS Journals Ltd 1999.

ABSTRACT: Bronchoscopic evaluation of stenosis is limited due to radial distortion of bronchoscopic images and the unknown distance between the endoscope and the stenotic area. The purpose of this study was the development and validation of a method for measuring cross-sectional areas in large airways.

Distance measurements were performed using a laser probe inserted into the working channel of a bronchoscope. The laser probe was positioned to the locus of interest in the airway, a ring of light (helium/neon) projected on to the luminal wall and the images acquired using an electronic bronchoscope. The images taken were distortion-corrected by means of a computer program. The method was validated by simulating airways using tubes of known diameter. Additionally, distortion-corrected bronchoscopic images were compared with distortion-free videoscopic image analysis of tracheal slices taken from pigs.

In the case of the plastic tubes, Pearson's correlation coefficient $(r)$ as well as the intraclass correlation coefficient (ICC) were slightly higher $(\mathrm{r}=0.99, \mathrm{p}<0.01, \mathrm{ICC}=0.97)$ than the correlation of cross-sectional areas between bronchoscopic and videoscopic images of tracheal slices $(r=0.88, p<0.01, \mathrm{ICC}=0.87)$.

This concept allows accurate and reproducible determination of cross-sectional areas in large airways.

Eur Respir J 1999; 14: 783-788.

Stenoses of the large airways may lead to severe dyspnoea, inspiratory stridor and cyanosis. Causes of tracheobronchial stenoses are often tumorous diseases and more benign processes such as postintubation stenosis, which may result in pneumological emergency situations. Today bronchoscopy is the technique of choice for the evaluation of large airways. Until now, however, no system has been successfully employed for the bronchoscopic measurement of airway stenosis.

Two major problems need to be solved in order to permit the endoscopic measurement of tracheobronchial stenosis. First, bronchoscopic images suffer from spatial distortion due to the wide-angle design of the endoscope lens. Due to this distortion, images are presented in circular (fish-eye) form. Thus, parts in the centre of an image captured using a wide-angle lens are presented as enlarged, and parts on the periphery appear smaller (fig. 1). Secondly, quantitative area determination requires information on the distance between the endoscope and the stenotic area.

The purpose of this study was to develop and to evaluate a bronchoscopic method for the measurement of crosssectional areas.

\section{Methods}

The endoscopic evaluation system presented here combines laser-based distance measurement with an imageprocessing technique for the correction of endoscopic image distortion. The bronchoscopic unit consists of an
Humboldt University, Charité, "Division of Pneumology, Dept of Internal Medicine I, *Clinic of Surgery **Outpatient Clinics of Internal Medicine ${ }^{+}$Dept of Radiology Institute of Medical Biometry, Berlin, Germany

Correspondence: C. Witt, Division of Pneumology, Dept of Internal Medicine I, Charité, Schumannstr. 20-21, 10098 Berlin, Germany. Fax: 493028023996

\section{Keywords: Airway evaluation} computer-assisted morphometry image-processing

Received: May 261997

Accepted after revision May 151999

Supported by the Colt Foundation
Olympus BF 1 T 200 electronic bronchoscope (Olympus Optical Co. Ltd., Tokyo, Japan) and an EVIS-CV 200 video processor (Olympus). The images were digitally input

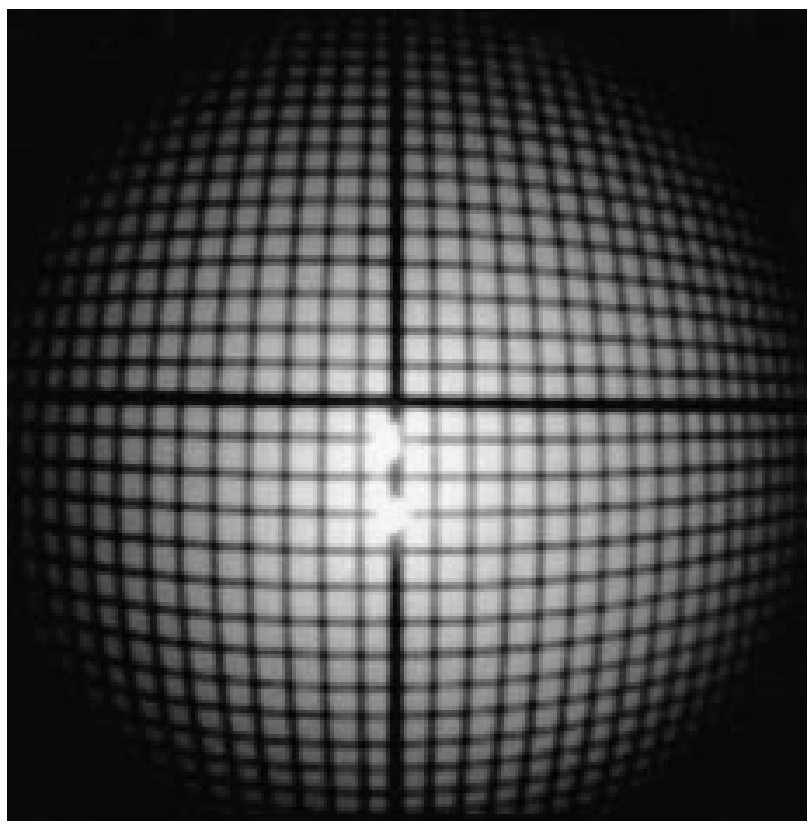

Fig. 1. - Distortion of an endoscopic image of millimetre-grid graph paper obtained using a BF 1 T 200 Olympus video endoscope. 
into a 486 DX 33 MHz IBM (IBM, New York, NY, USA) personal computer by a Screen Machine II Framegrabber Card (Fast Electronic, Munich, Germany).

The images obtained from the endoscope underwent morphometric analysis by means of a computer program developed by the authors to correct the distortion of the wide-angle lens. The distortion of a wide-angle lens is a function of radial distance from the centre of the image. This function is nonlinear. A co-ordination transformation was used to correct the distortion of the images captured by a wide-angle lens (fig. 2). If "x" and " $y$ " are the "real" coordinates of an object-point and " $\mathrm{x"}$ and " $\mathrm{y}$ " are the coordinates of the same point on the distorted image, a correction of the position is necessary in order to provide an undistorted picture (fig. 3). The correction factors for the two directions $\left(x-x^{\prime}\right.$ and $\left.y-y^{\prime}\right)$ depend on the radial distance from the centre of the image. To apply these correction factors to the image, the generation of a coordination matrix is necessary. Millimetre grids were used to assess the distortion by measuring the positions of grid points relative to the central one. The co-ordinates of the imaged grid points were digitized from the computer screen. After this process had been performed for each grid point in the distorted image, the radial distortion correction matrix was completed. Contrast, brightness and colour saturation were not changed when applying the distortion correction program.

A laser probe inserted into the operating channel of the bronchoscope enabled assessment of the distance between the images and the tip of the bronchoscope. As radiation source the helium/neon pilot of a neodymiumyttrium aluminium garnet (YAG) laser (with output power rating of $1 \mathrm{~mW}$ ), routinely applied in bronchological laser treatment, was used. The laser probe consisted of a hard clad saliva (HCS) fibre with a $400-\mu \mathrm{m}$ core diameter and a numerical aperture of 0.18 . A reflector was attached to the top of the fibre, which enabled reflection of the laser beam at an angle of $90^{\circ}$ (fig. 4). Laser fibre and reflector were con-

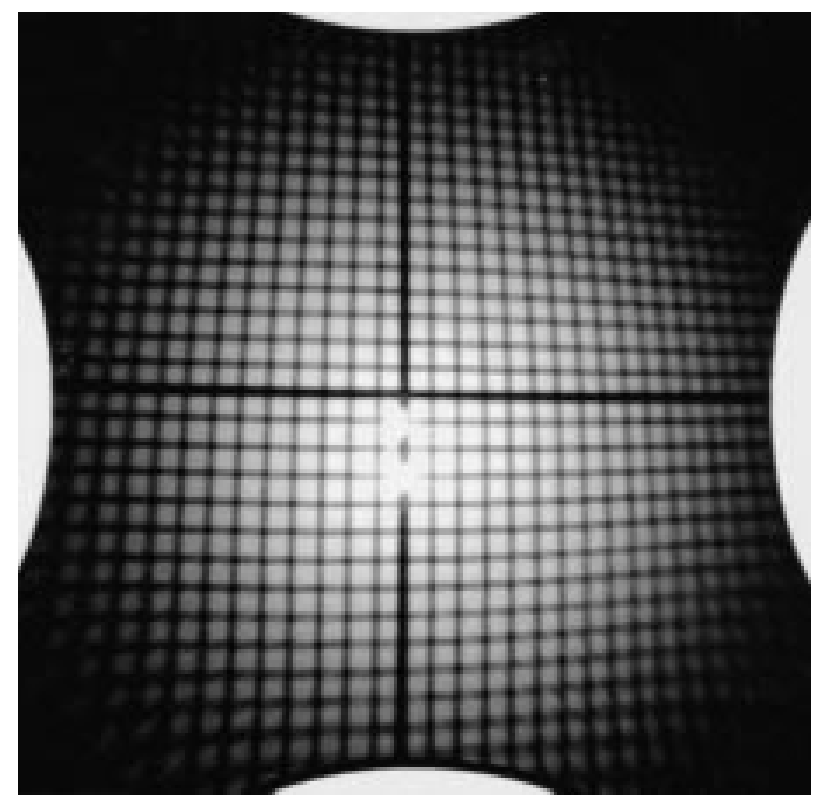

Fig. 2. - The same image as shown in figure 1 after distortion correction by the distortion-correction program. Note that the best correction is obtained in the centre of the image.

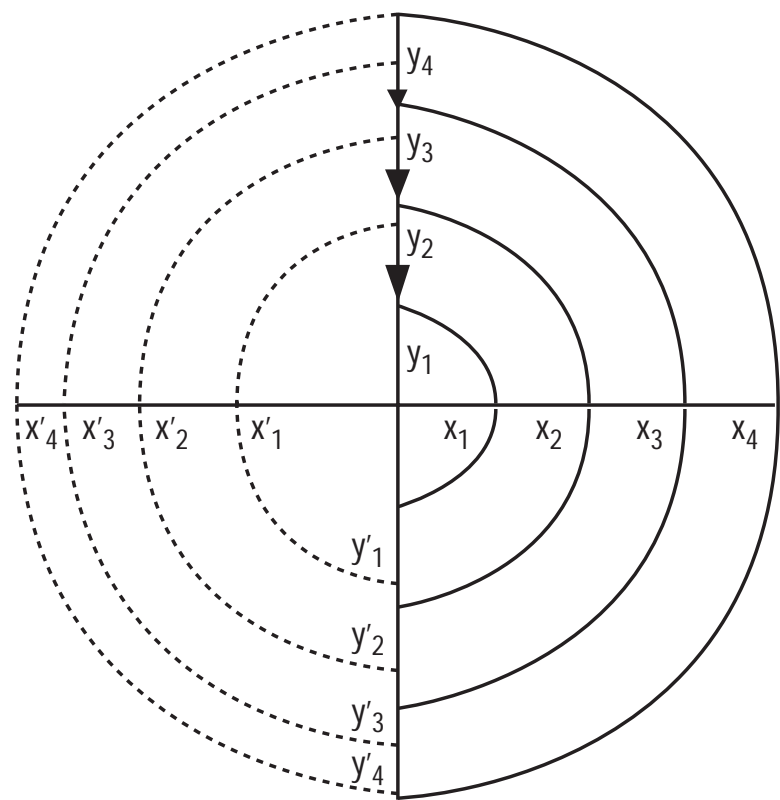

Fig. 3. - Example of a reconstruction of an image. The co-ordinates of the undistorted object are $\mathrm{x}$ and $\mathrm{y}$, and the co-ordinates of the distorted image are $x^{\prime}$ and $y^{\prime}$. The right half of the figure shows the original positions of four circles equidistant from each other. The distorted image is presented in the left half. Note that the spacing between the circles has changed. Whereas the distance to the points lying on innermost circle has been markedly, increased, the distances between the outer circles have decreased. Arrows symbolize the effects of distortion correction by applying a co-ordination transformation via computerized image processing. The points of the image $\left(\mathrm{x}^{\prime}, \mathrm{y}^{\prime}\right)$ are transformed through a nonlinear relation to the corresponding points of the object $(\mathrm{x}, \mathrm{y})$.

nected by means of a transparent catheter covering the laser probe and the reflector. The distance between the reflector and the end of the laser fibre was only $0.5 \mathrm{~mm}$ and the connection was fixed to maintain the optical axis of the system.

The laser probe projects a ring of light on to the endoluminal wall. The laser probe can shift relative to the bronchoscope in the operating channel. A marker on the surface of the laser probe shows its position relative to the bronchoscope. Thus, a distance of $15 \mathrm{~mm}$, between the plane of the projected laser light ring and the tip of the bronchoscope could be obtained during all examinations. Because of the known distance between the endoscopic

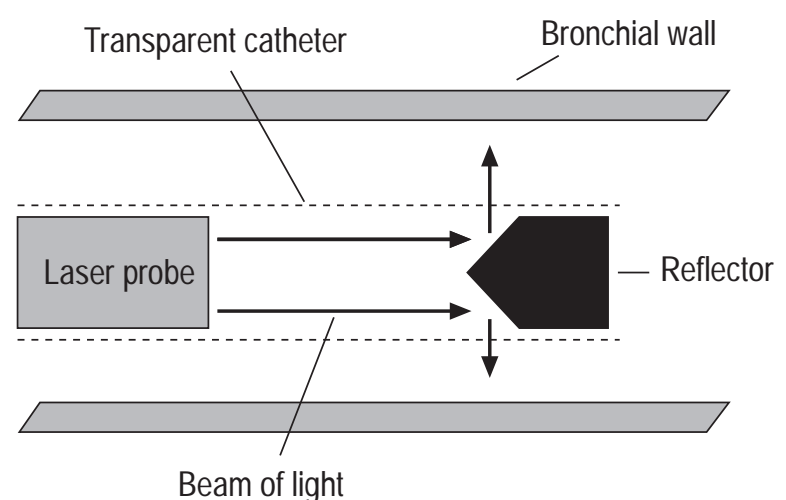

Fig. 4. - Schematic diagram of the beam of light using the laser probe. 


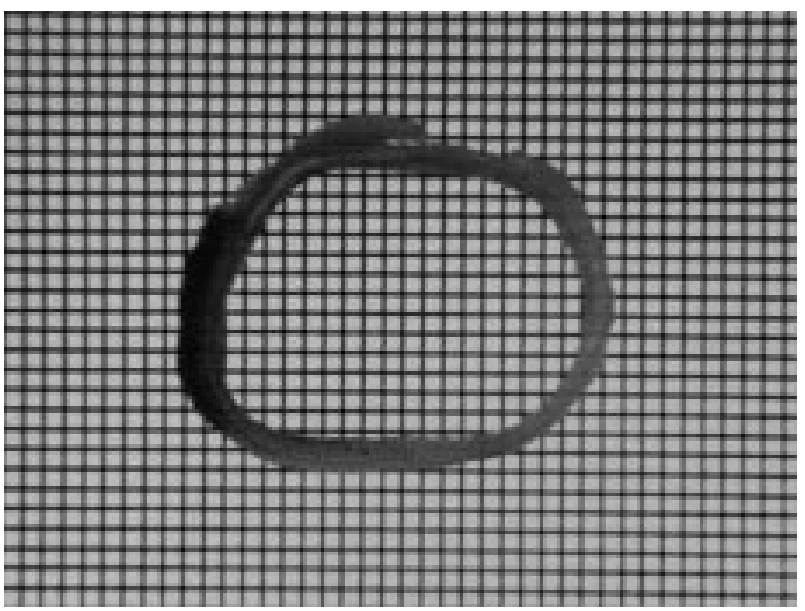

Fig. 5. - Slice of a tracheal ring placed on millimetre-grid graph paper. The image was obtained using the video macro zoom lens. Note that the image is distortion-free.

lens and the reflector, it was possible to morphometrically analyse the area within the ring of light using a computer image analyser. The computer program NIH (National Institutes of Health, Bethesda, MD, USA) Images, version 1.6, was used for area measurement. This programme was run on an Apple Performer 5400 Computer (Apple, Cupertino, CA, USA). Before assessment of the areas, standardization was necessary in order to define the relationship between the number of pixels and the area in $\mathrm{mm}^{2}$. An image was therefore obtained from a millimetre grid from the same distance $(15 \mathrm{~mm})$ as used between reflector and bronchoscope tip. After correction of endoscopic image distortion, it was possible to determine the area of a certain structure after comparison with the original image.

Plastic tubes and models of the bronchial system with known luminal diameters were initially used for experimental endoscopic studies. The diameters of the plastic tubes ranged $6.2-17.2 \mathrm{~mm}$. The plastic tubes were white and made of polystyrene.

After verifying the accuracy of the determination of the diameter of plastic tubes with round cross-sectional areas, necropsy specimens of pig trachea were used to simulate more realistic conditions. Three tracheae were used after fixation in formaldehyde (10\% solution). Tracheae were subsequently conserved in polyethylene glycol in order to preserve the shape of the tracheal rings after dissection. Pig trachea images were obtained at $2-\mathrm{mm}$ intervals. After completion of endoscopy, the tracheae were dissected and the cross-sectional areas of the tracheal rings (thickness 2 $\mathrm{mm}$ ) were determined using a video macro zoom lens (18108 mm; Fuji Photo Film Co. Ltd., Tokyo, Japan) and a WVE 550 3-IT-CCD digital processing camera (Panasonic, Matsushita Electric Industrial Co. Ltd., Osaka, Japan). This lens provides almost distortion-free images of objects (fig. 5). Bronchoscopic image measurements were compared with videoscopic image measurements. The images were obtained by means of the technique described above.

Area measurements of tubes and pig's tracheae were made by three investigators and were performed twice by each investigator in order to assess reproducibility and interobserver variability. Comparisons of the bronchoscopic and radiological determinations of the smallest airway area were performed in four patients. All patients suffered from tracheal stenosis following intubation. Computed tomography (CT) was performed using a commercially available helical CT scanner (Tomoscan 7000; Phillips Medical Systems, Eindhoven, the Netherlands). Images were acquired at a slice thickness of $1.5 \mathrm{~mm}$ and an increment of $2 \mathrm{~mm}$, and were reconstructed at a resolution of $1 \mathrm{~mm}(50 \mathrm{~mA}, 120 \mathrm{kV}, 1$-s scan, matrix $512 \times$ $512)$. For evaluation, a standard soft tissue setting (400/50) was used.

\section{Statistics}

The association between two measuring methods as well as between two observers were each evaluated by the intraclass correlation coefficient (ICC), expressing reproducibility and inter-observer variability, respectively. In addition, linear regression was used to analyse the relationship between real areas and measurements made by the proposed method (response). The intercept, slope, Pearson's correlation coefficient and corresponding confidence regions for single as well as average predictions were obtained from the regression lines.

\section{Results}

Insertion of the laser probe into the operating channel of the bronchoscope enabled a ring of light as projected on to the inner wall of the plastic tubes and the trachea to be viewed. It was possible to detect the ring of laser light in every image (fig. 6).

Use of the distortion-correction program enabled almost complete elimination of optical distortion (fig. 2). Distortion remained only at the periphery of the image. When plastic tubes with known luminal diameters were used as a model, the correlation coefficient (r) reached 0.99 $\left(\mathrm{p}<0.01, \mathrm{ICC}=0.97\right.$ (measured area (in $\left.\mathrm{mm}^{2}\right)=6.47+$

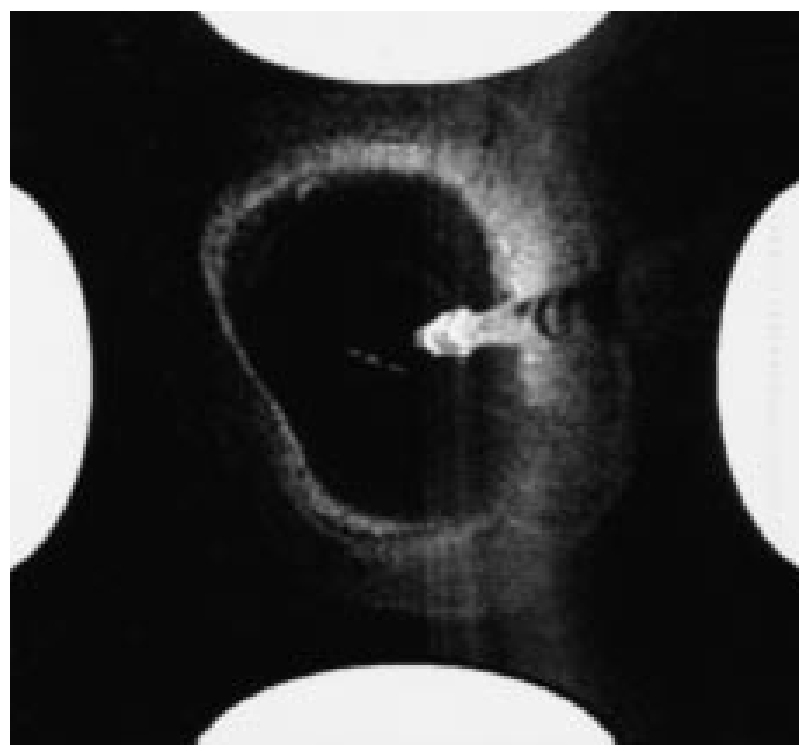

Fig. 6. - Image of a pig trachea after distortion correction by applying the distortion-correction program and before cutting the trachea. The laser probe projecting the ring of light is seen. 


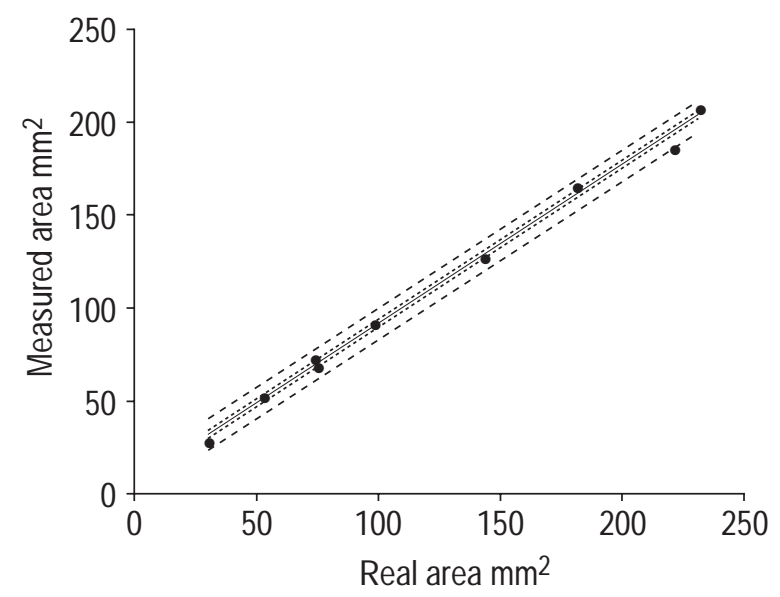

Fig. 7. - Validation of the method using plastic tubes (of diameter 6.2, $8.2,9.7,9.8,11.2,13.1,15.2,16.8$, and $17.2 \mathrm{~mm}$ ). Data were obtained at a distance of $15 \mathrm{~mm}$ from the bronchoscope. The real areas were calculated from the known radius. The measured areas were obtained after distortion correction and morphometry. - - - : the confidence limit for an average prediction; prediction $(\mathrm{r}=0.99, \mathrm{p}<0.01)$.

$(0.85 \times$ real area $)))$. Figure 7 shows the small difference between the real and calculated area.

The measurements of areas within the pig tracheal rings using the macro zoom lens ranged $197.4-239.5 \mathrm{~mm}^{2}$. The same areas measured using the distortion-correction algorithm ranged 196.6-238.6 mm $\mathrm{mm}^{2}$ An $\mathrm{r}$ of $0.88(\mathrm{p}<0.01$, ICC $=0.87$ (measured area $\left(\right.$ in $\left.\mathrm{mm}^{2}\right)=19.70+(0.92 \times$ real area))) resulted from comparison of bronchoscopic image measurements with videoscopic image measurements (fig. 8 ). Comparisons between bronchoscopic determination of the smallest area of stenosis and the results of computed tomography are expressed in table 1. Except for one patient, bronchoscopically determined stenotic areas tended to be slightly smaller than those obtained by means of CT.

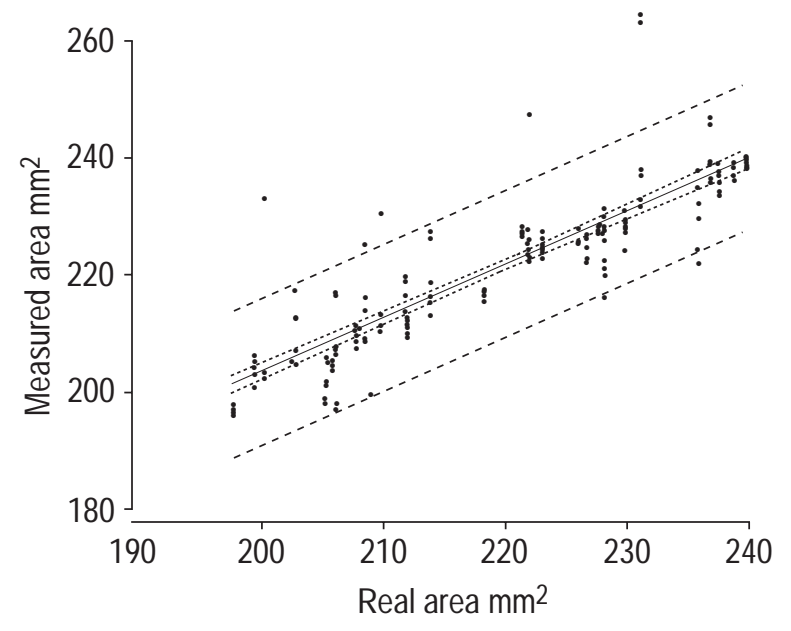

Fig. 8. - Comparison between videoscopic (video macro zoom lens) and bronchoscopic area measurements of tracheal slices. The real areas of the tracheal slices were measured by means of a distortion-free macro zoom video lens. The measured areas were obtained from the same tracheal slice using distance-related distortion correction. ---- : the confidence limit for an average prediction; 1 : the confidence limit for a single prediction $(r=0.87, \mathrm{p}<0.01)$.
Table 1. - Comparisons of bronchoscopic and radiological computed tomography (CT) measurement of the smallest area of tracheal stenosis

\begin{tabular}{lcc}
\hline & \multicolumn{2}{c}{ Means of measurement } \\
\cline { 2 - 3 } Patient No & Bronchoscopy $\mathrm{mm}^{2}$ & $\mathrm{CT} \mathrm{mm}$ \\
\hline 1 & 113.1 & 98.3 \\
2 & 51.8 & 54.2 \\
3 & 24.6 & 34.8 \\
4 & 51.4 & 58.6 \\
\hline
\end{tabular}

Within the context of the above small number of patients, the preliminary results of bronchoscopic area determination showed a trend toward slight underestimation of the smallest stenotic area.

Electronic bronchoscopes are more expensive than conventional flexible ones. Therefore, comparative measurements were also performed. Likewise, various flexible bronchoscopes (Olympus BF 1 T 200, BF P 30, BF T 30, and BF $20 \mathrm{D}$ ) were studied. This was done in a fashion identical to that used for the recording of the millimetre grids for calibration of the distortion-correction algorithm. The area of a square in pixels and the distance from the centre of the image (radius) in $\mathrm{mm}$ was recorded for each bronchoscope (fig. 9). It was possible to perform distortion correction with every flexible bronchoscope, but different lenses required different distortion correction factors. An electronic bronchoscope was used for the final validation because of its better optical resolution.

\section{Discussion}

Determination of airway size is often required in bronchology. A new technique has been presented for endoscopic solution of this problem. The characteristics of this new technique include distortion correction of bronchoscopic images as well as distance measurement using the projection of laser light on to the inner wall of the airway. Application of this system allows direct measurement of airway lumina.

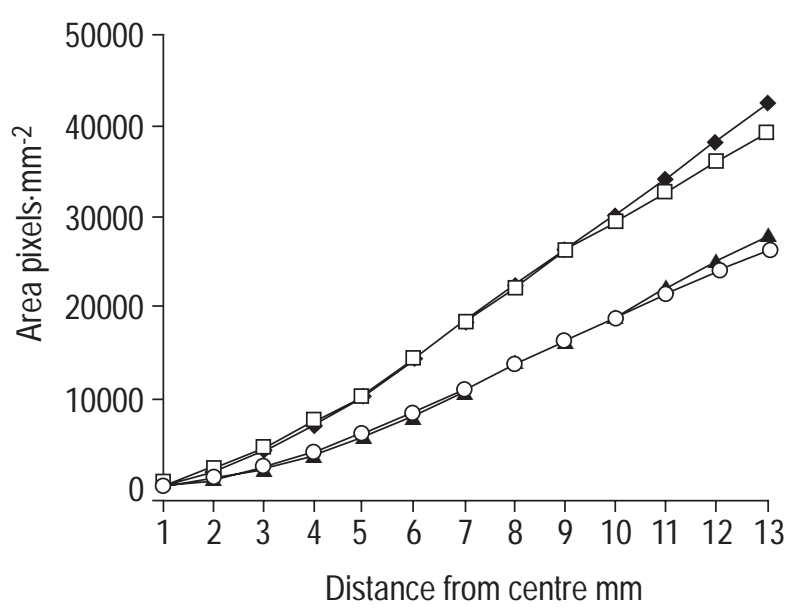

Fig. 9. - Area measurements of squares (on a millimetre grid) in pixels viewed through various bronchoscopes and distances from the centre of the image. All images were recorded with a distance of $15 \mathrm{~mm}$ between the bronchoscope and the millimetre grid. $\boldsymbol{\Delta}$ : BF 1 T 200; $\diamond$ : BF 20 D; $\square$ : BF T 30; O: BF P 30. The bronchoscopes BF 1 T 200 and BF 20 D yielded larger areas than the BF T 30 and the BF P 30 because of their different objective lenses. 
There are various methods for measuring stenoses of trachea or main bronchi. Methods include measurement of lung function, radiographic imaging, endoscopy and analysis of sound [1-5]. The main disadvantage of these methods is the indirect manner of measurement. Only the technique of bronchoscopy enables direct visualization of the stenotic region. Measurements of airway resistance or maximal expiratory flow reflect the functional rather than the anatomical severity of obstruction, and provide no information concerning the size of the airways. Therapeutic options for central airway stenosis, however, are based on the length and severity of the stenosis. For example, in cases of malignant stenosis, laser therapy is used for exophytic tumours, whereas stenting, afterloading therapy or a combination of therapeutic techniques is required for large extended endoluminal tumours [6-10]. These circumstances emphasize the need for a system enabling exact measurement of the severity of stenosis, as well as its direct visualization.

Radiographic findings, especially $\mathrm{CT}$ in combination with multiplanar reconstruction, can accurately establish the location and degree of central airway stenosis [11]. Recently, initial reports published on virtual bronchoscopy by means of CT have offered new possibilities for quantitative area determination [12, 13]. One major disadvantage of all radiographic methods, however, is the use of x-rays. In addition, endoscopic measurement of airway stenosis is diagnostically more valuable. Colour information, for instance, is required for the assessment of endobronchial inflammation, the size of tumours and the risk of haemorrhage. Furthermore, secretory products such as mucus and blood or necroses must often be removed before accurate data can be obtained.

Additionally, information gained from flexible bronchoscopic examination relates to the dynamics of vocal-cord function and enables diagnosis of tracheal collapse. With the use of high-resolution CT, the severity of tracheomalacia is frequently underestimated due to the lack of available dynamic information. Conversely, based on the results of cine-CT (low-resolution images), focal stenoses of the trachea can be interpreted as areas of variable narrowing due to craniocaudal movement of the trachea during respiration [14].

Only bronchoscopy enables reliable diagnosis of dynamic airway stenosis. Application of the present method could offer quantitative assessment of the degree of dynamic airway collapse and tracheobronchomalacia. An endoscopic technique with the ability to directly measure and quantify airway diameter could accordingly provide essential information concerning airway stenosis. Additionally, a reproducible classification system of tracheobronchial stenoses on the basis of endoscopy would provide the basis for comparison of the results obtained using various techniques for treating this condition.

Estimation of the severity of airway stenosis as \% stenosis, by comparing normal proximal or distal airway size, often remains the only available basic endoscopic technique for determining the need for therapeutic intervention [1]. The estimate of the degree of severity often varies from examiner to examiner. Moreover, airway resistance depends on the length of the stenosis. McCAFFrey [15] has proposed a classification system for laryngotracheal ste-noses, including information on the location and length of the stenosis. This classification into four stages of stenosis has been shown to effectively predict the likelihood of successful decannulation.

Using the present method, information on the length of the stenosis is obtained by repeated measurements of crosssectional areas in combination with external distance measurement (for example, distance between tooth and tip of bronchoscope).

Images obtained through an endoscope are distorted: the wide-angle lenses used in endoscopes produce a "barrel" effect. Areas in the image at the perimeter of the field of view appear smaller than they actually are. This change in size is nonlinear and precludes quantitative measurement [16].

Various authors have developed and tested correction procedures for endoscopic images [16-18]. None of the methods dealing with assessment of airway size by means of distortion correction programs, however, have been able to be used in vivo without knowledge of the distance between the objective lens and the object. Studies dealing with endoscopic measurement of lesion sizes have, therefore, often required the insertion of bodies of known diameter (biopsy forceps, marker bodies, etc.) into the lesion [17-19].

FORKERT et al. [19] accurately calculated correction factors for assessment of airway calibre as a function of the size and position of the region of interest in the image. A quantitative comparison of videobronchoscopic and CT measurements was performed in an animal model and revealed similar changes in airway diameter during vagal stimulation. For the purposes of calibration, the authors used a marker ball inserted into the airway before measurement. In the authors' opinion, such a requirement for the application of calibration bodies limits the opportunities for airway measurements in humans.

Other investigations were performed only on models, and enabled direct measurement of the distance from bronchoscope [20]. In the present study, the accuracy of distortion correction in combination with laser-based distance measurements was verified by means of in vitro investigation of animal models. The first results, in four patients, disclosed acceptable correlation with CT, thereby indicating justification of its further use in patients on a routine basis. The method is safe and inexpensive and allows images to be obtained immediately during bronchoscopic examination.

There is no need for the exclusive use of monochromatic laser light. Laser light was used in order to minimize divergence. Application of this system is also possible with white light, which reduces costs.

The ICC of 0.97 for the plastic tubes and 0.87 for the tracheal slices, respectively, demonstrate acceptable reproducibility and interobserver variability of two area measurements performed by three investigators [21].

A disadvantage of the method presented is that the image of the projected ring cannot be completely seen. Because the laser probe with the reflector is located in the centre of the image, a small part of the ring must be extrapolated. Enhancement of this technique is therefore necessary. The smaller the diameter of the laser probe, the smaller the missing part of the ring. The direct nature of the visualization enables correction of the endoscope if part of the ring is not optimally visualized for cases of irregular wall structures or ridges. The divergence of the reflected 
laser beam additionally allows an increase in the width of the laser ring, resulting in turn in enhanced visualization.

Erroneous findings result if the laser probe deviates from the axis of the bronchoscope. On the assumption that AC is a circle encompassing the true area, $\mathrm{AE}$ is an ellipse encompassing the resulting "incorrect" area and $\alpha$ is the angle between the axis of the bronchoscope and the laser probe, the following equations enable estimation of the relative error: $\mathrm{AE} / \mathrm{AC}=\mathrm{x} ; \mathrm{x}=\cos \alpha$; and relative error $=1-\mathrm{x}$. Therefore relative error $=1-\cos \alpha$. The resulting error $(\mathrm{x})$ is $0.38 \%$ for $5^{\circ} 1.52 \%$ for $10^{\circ}, 3.4 \%$ for $15^{\circ}, 6.03 \%$ for $20^{\circ}, 9.37 \%$ for $25^{\circ}, 13.4 \%$ for $30^{\circ}$ and $29.2 \%$ for $45^{\circ}$. If the endoscope with the laser probe is not inserted in the direction of the long axis of the airway, overestimation of cross-sectional area results. Thus, repeated measurements are proposed for the determination of the smallest cross-sectional area in order to alleviate these problems. It is not essential that the laser probe is positioned centrically or eccentrically.

In cases of very small cross-sectional stenotic areas, passage of the bronchoscope may be dangerous. The contact of the bronchoscope with the endobronchial wall may lead to aggravation of the severity of the stenosis due to inflammatory reaction. A further advantage of the method described above is that it does not require contact with the surface of a stenosis.

Application of the developed system enables direct documentation of airway size and shape. In cases of malignant or benign stenoses, this technique is of great diagnostic value due to the possibility of accurately measuring the degree of stenosis. These benefits allow determination of the need and optimal time for intervention. Additionally, this new method may prove beneficial in therapeutic bronchoscopy, e.g., for work prior to stent implantation, choice of stent diameter or follow-up of interventional bronchoscopic techniques. Further investigations are necessary to determine the diagnostic accuracy on a large scale in patients with various types of tracheobronchial stenoses.

\footnotetext{
Acknowledgements. The authors thank $\mathrm{H}$. Laufer (video studio, Charité Medical Center) for his technical assistance.
}

\section{References}

1. Cotton RT. Pediatric laryngotracheal stenosis. J Pediatric Surgery 1984; 19: 699-704.

2. Vossing M, Wassermann K, Eckel HE, Ebeling O. Peak flow measurements in patients with laryngeal and tracheal stenoses. A simple and valuable spirometric method. HNO 1995; 43: 70-75.

3. Rothschild MA. The airway scale: a simple tool for comparing calibers of airway devices. Otolaryngol Head Neck Surg 1994; 11: 847-848.
4. Yonemaru M, Kikuchi K, Mori M, et al. Detection of tracheal stenosis by frequency analysis of tracheal sounds. J Appl Physiol 1993; 75: 605-612.

5. Czaja JM, McCaffrey TV. Acoustic measurement of subglottic stenosis. Ann Otol Rhinol Laryngol 1996; 105 : 504-509.

6. Personne CA, Colchen A, Leroy M, Vourch G, Toty L. Indications and technique for endoscopic laser reactions in bronchology. A critical analysis based upon 2,234 resections. J Thorac Cardiovasc Surg 1986; 91: 710-715.

7. Becker HD. Stenting the central airways. J Bronchology 1995; 2: 98-106.

8. Colt HG, Dumon JF. Airway obstruction in cancer: The pros and cons of stents. J Respir Dis 1991; 12: 741-749.

9. Speiser BG, Spratlin L. Remote afterloading brachytherapy for local control of endobronchial carcinoma. Int J Rad Oncol Biol Phys 1993; 25: 589-597.

10. Witt D, Zietz G, Petri F, Ewert R, Baumann G. Flexible bronchoscopic stenting followed by radiation in malignant obstruction of central airways. J Bronchology 1995; 2: 118-222.

11. Whyte RI, Quint LE, Kazerooni EA, Cascade PN, Iannettoni MD, Orringer MB. Helical computed tomography for the evaluation of tracheal stenosis. Ann Thorac Surg 1995; 60: 27-30.

12. Summers RM, Feng DH, Holland SM, Sneller MC, Shelhamer JH. Virtual bronchoscopy: segmentation method for real-time display. Radiology 1996; 200 : 857-862.

13. Vining DJ, Liu K, Choplin RH, Haponik EF. Virtual bronchoscopy. Relationships of virtual reality endobronchial simulations to actual bronchoscopic findings. Chest 1996; 109: 549-553.

14. Brody AS, Kuhn JP, Seidel FG, Brodsky LS. Airway evaluation in children with use of ultraflast CT: pitfalls and recommendations. Radiology 1991; 178: 181-184.

15. McCaffrey TV. Classification of laryngotracheal stenosis. Laryngoscope 1992; 102: 1335-1340.

16. Smith WE, Vakil N, Maislin SA. Correction of distortion endoscope images. IEEE Trans Med Imaging 1992; 11: 117-122.

17. Kouwenhoven E, Mast F, van Rijk-Zwikker GL. Geometrical reconstruction of images obtained with electronic endoscopy. Phys Med Biol 1993; 38: 13-24.

18. Vakil N, Smith W, Bourgeois K, Everbach EC, Knyrim K. Endoscopic measurement of lesion size: improved accuracy with image processing. Gastrointest Endosc 1994; 40: 178-183.

19. Forkert L, Watanabe H, Sutherland K, Vincent S, Fisher JT. Quantitative videobronchoscopy: a new technique to assess airway caliber. Am J Respir Crit Care Med 1996; 154: 1794-1803.

20. Santos MC, Strande L, Doolin EJ. Airway measurement using morphometric analysis. Ann Otol Rhinol Laryngol 1995; 104: 835-838.

21. Guggenmoos-Holzmann I, Wernecke K-D. Medizinische Statistik. Blackwell Wissenschaftsverlag, Berlin, Wien, 2.Auflage, 1998. 